\title{
First-line tracheal resection and primary anastomosis for postintubation tracheal stenosis
}

\author{
H Elsayed, AM Mostafa, S Soliman, T Shoukry, AA El-Nori, HY El-Bawab
}

\author{
Ain Shams University Hospital, Cairo, Egypt
}

\section{ABSTRACT}

INTRODUCTION Tracheal stenosis following intubation is the most common indication for tracheal resection and reconstruction. Endoscopic dilation is almost always associated with recurrence. This study investigated first-line surgical resection and anastomosis performed in fit patients presenting with postintubation tracheal stenosis.

METHODS Between February 2011 and November 2014, a prospective study was performed involving patients who underwent first-line tracheal resection and primary anastomosis after presenting with postintubation tracheal stenosis.

RESULTS A total of 30 patients (20 male) were operated on. The median age was 23.5 years (range: 13-77 years). Seventeen patients $(56.7 \%)$ had had previous endoscopic tracheal dilation, four $(13.3 \%)$ had had tracheal stents inserted prior to surgery and one (3.3\%) had undergone previous tracheal resection. Nineteen patients $(63.3 \%)$ had had a tracheostomy. Eight patients (26.7\%) had had no previous tracheal interventions. The median time of intubation in those developing tracheal stenosis was 20.5 days (range: 0-45 days). The median length of hospital stay was 10.5 days (range: 7-21 days). The success rate for anastomoses was $96.7 \%$ (29/30). One patient needed a permanent tracheostomy. The in-hospital mortality rate was 3.3\%: 1 patient died from a chest infection 21 days after surgery. There was no mortality or morbidity in the group undergoing first-line surgery for de novo tracheal lesions.

CONCLUSIONS First-line tracheal resection with primary anastomosis is a safe option for the treatment of tracheal stenosis following intubation and obviates the need for repeated dilations. Endoscopic dilation should be reserved for those patients with significant co-morbidities or as a temporary measure in non-equipped centres.

\section{KEYWORDS}

Stenosis - Postintubation injury - Tracheal resection

Accepted 23 August 2015

\section{CORRESPONDENCE TO}

Hany Elsayed, E: drhany.elsayed@yahoo.co.uk

In the vast majority of patients, acquired stenosis of the subglottis and trachea is a direct result of prolonged intubation. ${ }^{1}$ On the whole, this is due to the increased use of mechanical ventilation and endotracheal intubation in intensive care units, and may in part explain the growing rate of laryngotracheal stenosis in patients following intubation $(0.6-21 \%) .^{2}$ Following the work of Pearson and Andrews in the $1970 \mathrm{~s},{ }^{3}$ complete resection of the stenotic segment with primary anastomosis has been accepted as the procedure of choice, a result of its high success rate $(71-95 \%)^{1,2,4,5}$ and minimal morbidity. Other methods for treating benign tracheal stenosis (such as endoscopic dilation and stenting) are still being used but have not always proved as effective. ${ }^{2,4}$

In this study, first-line tracheal resection and primary anastomosis was performed as the principal method of treatment for postintubation tracheal stenosis, without using other modalities of treatment and (when possible) before a tracheostomy was needed for urgent airway obstruction. The outcome and effectiveness of this policy was evaluated.

\section{Methods}

A prospective study was performed between February 2011 and November 2014 involving patients who had benign postintubation tracheal stenosis. Inclusion criteria consisted of patients who underwent first-line tracheal resection and primary anastomosis. This included patients who had dilations, stents and tracheostomies in other centres before presenting to us. It also included patients with de novo tracheal lesions who had had no previous interventions. Patients with postintubation glottic stenosis not amenable to surgery were excluded from the study, as were patients who had had tracheal stents or tracheostomies as definitive management because of significant co-morbidities. All patients were operated on in Ain Shams University Hospital, which is the only tertiary centre for tracheal surgery in Egypt. Four surgeons operated on all the patients using the same technique.

\section{Preoperative assessment}

Patients included in the study had a history of tracheal stenosis and some had had previous endoscopic dilations or 
tracheal stents with or without tracheostomies. All patients presenting with stridor and/or respiratory difficulty are assessed clinically following a direct referral or following review in the ear, nose and throat (ENT) department. Computed tomography (CT) of the neck and chest is a valuable preoperative imaging modality, with volume rendering reconstruction of the airway performed when feasible (Fig 1). Patients are examined routinely using direct laryngoscopy to assess vocal cord mobility and to exclude glottic stenosis. Patients presenting with acute airway obstruction and needing urgent intervention are transferred to the cardiothoracic department on an emergency basis and operated on immediately.

\section{Operative intervention}

The patient is typically placed in a supine position with the arms tucked against the body and the neck extended. In order to help with neck extension, an inflatable ('thyroid') bag is placed under the shoulders. A nasogastric tube is inserted. Operations are preceded by careful endoscopic evaluation of the airway, using the smallest rigid bronchoscope possible. If there is total lumen obstruction (grade 4 stenosis), we rely on the preoperative CT.

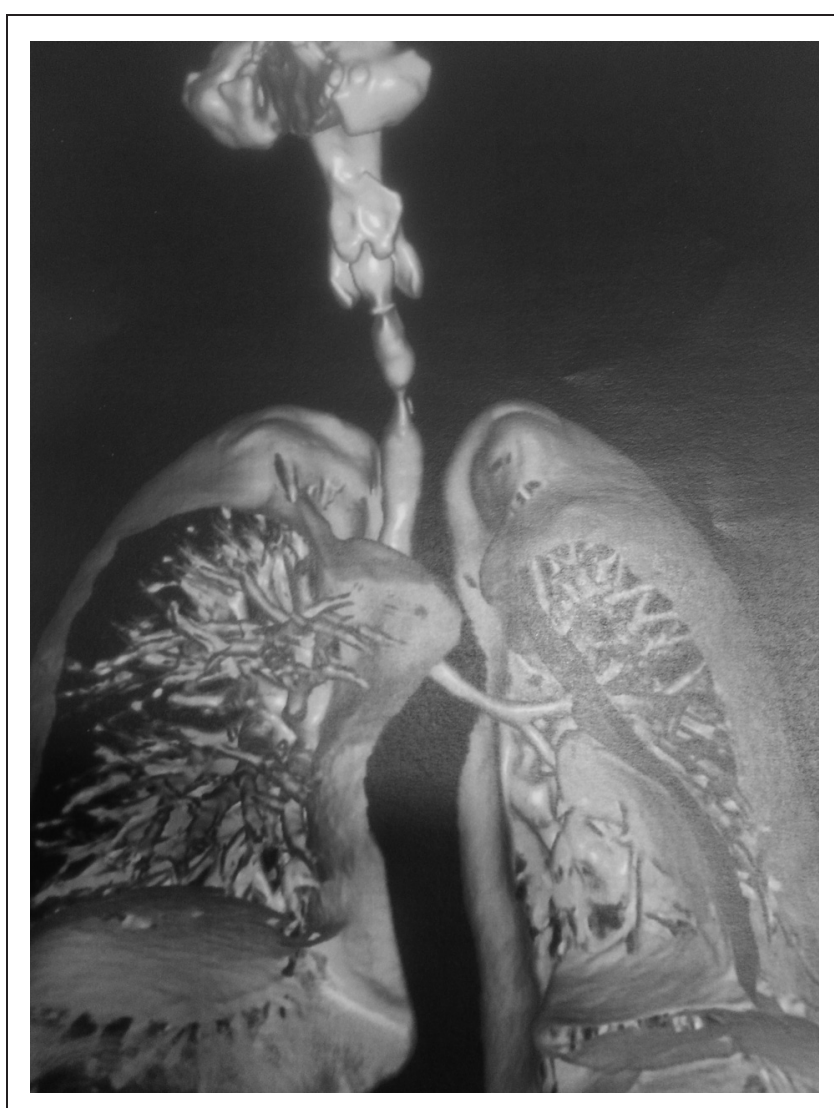

Figure 1 Volume rendering computed tomography of the neck and chest showing midtracheal stenosis
Two distinct parameters are assessed and communicated explicitly by the surgeon to the rest of the theatre team before resection begins: the location and extent of the lesion to be resected, and coexistence of tracheomalacia. If the lesion is less than half the tracheal length and there is no glottic stenosis (and the distal tracheal mucosa is healthy), the lesion is considered resectable. The patient is then intubated with the largest endotracheal tube (ETT) possible. A tight stenosis can be dilated at this time to allow endotracheal intubation and to avoid accumulation of carbon dioxide during the intervention.

Proximal tracheal lesions are approached via a transverse cervical incision. Skin flaps are raised. The trachea is dissected sharply and mobilised, ensuring the blood supply and the recurrent laryngeal nerve (RLN) that lies laterally are not jeopardised. Bipolar diathermy is used to reduce the chance of thermal injury to the RLN. More distal tracheal lesions are approached via a combined cervical and manubriotomy approach. Only when long segment tracheal stenosis is present or in cases of carinal stenosis is a full sternotomy performed. A suprahyoid release or hilar release with division of the inferior pulmonary ligament is planned according to the site and extent of the lesion.

Following complete transection of the trachea, an appropriately sized ETT is placed in the distal trachea, resuming ventilation. After resection, the posterior wall is anastomosed using interrupted absorbable sutures (polydioxanone). An oral ETT is then advanced beyond the anastomosis, which is completed in a similar interrupted fashion. No local antiproliferative agents are used.

After the trachea is closed, an anastomotic leak test is undertaken. This is done by flooding the operative field with saline, deflating the ETT cuff and applying $30 \mathrm{~cm}$ of water pressure to the breathing circuit. If no bubbles are seen, the incision is closed after placing a suction drain in the pretracheal space.

Before extubation, a 'guardian' suture is placed from the skin over the manubrium to the submental crease, to ensure that the neck remains flexed. All patients are extubated in a carefully controlled fashion. Particular attention to airway suctioning aims to prevent vigorous coughing, which might stimulate unwanted neck extension. Following extubation, care is taken to check for airway obstruction (which might suggest bilateral vocal cord paralysis) and hoarseness or aspiration (which may be caused by unilateral vocal cord paralysis). A distal tracheostomy is ready to be placed immediately (especially with corrected subglottic stenosis). This remains until another direct laryngoscopy and bronchoscopy are performed (usually after ten days). In cases of postoperative bilateral vocal paralysis, the patient is referred back to the ENT department for widening of the airway by cordotomy using radiofrequency ablation.

\section{Postoperative care}

Patients are transferred to an intensive care setting for careful observation for the development of any obstructive symptoms. Feeding is resumed on postoperative day 1 or 2 (beginning with liquids), paying attention to signs of aspiration. On the day after surgery, patients without 
complications can be transferred to a regular hospital ward, with experienced nursing care. Regular physiotherapy is essential as a mini-tracheostomy is not used routinely except in patients with unilateral vocal cord paralysis or a weak cough reflex. Patients are usually kept in the hospital for seven days. If no problems are identified, they are discharged home after the neck stitches have been removed.

\section{Follow-up review}

All patients are seen 3-4 weeks after discharge to check the progress of their recovery. Our patients are followed up by telephone a few months after the procedure with regard to breathing and quality of life. Any patients with dyspnoea, hoarseness, aspiration or stridor are subjected to a direct laryngoscopy and bronchoscopy to check the mobility of the vocal folds as well as the patency of the tracheal lumen. Those with unilateral vocal cord paralysis are referred to the ENT department.

All data were collected from patient records and telephone calls. Patients were consented to be enrolled in this study. Ethical committee approval was obtained to proceed with the study according to the university's policy.

\section{Results}

A total of 30 patients ( 20 male) were operated on during the study period. The median age was 23.5 years (range: $13-77$ years).

\section{Preoperative characteristics}

The median time of intubation causing tracheal stenosis was 20.5 days (range: $0-45$ days). All our patients had complex lesions $(>1 \mathrm{~cm})$. One patient $(3.3 \%)$ had grade 1 stenosis, three $(10.0 \%)$ had grade 2 , twenty-three $(76.7 \%)$ had grade 3 and three $(10.0 \%)$ had grade 4 . Twenty-two patients had had previous tracheal interventions: seventeen $(56.7 \%)$ had previous endoscopic tracheal dilation, four $(13.3 \%)$ had tracheal stents prior to surgery and one $(3.3 \%)$ had previous tracheal resection. Among the 22 patients who had had previous interventions, 19 ( 2 children and 17 adults, 63.3\%) had tracheostomies on presentation. Eight patients (26.7\%) had had no previous tracheal interventions and underwent firstline resection for de novo tracheal lesions.

\section{Operative findings}

A cervical incision was sufficient in 24 patients $(80.0 \%)$ while $3(10.0 \%)$ needed an additional manubriotomy and $3(10.0 \%)$ required a full sternotomy for resection of lesions (1 had a $6 \mathrm{~cm}$ lesion, 1 had a $6.5 \mathrm{~cm}$ lesion, and 1 needed the sternotomy to allow tracheal release manoeuvres to permit resection and anastomosis as this patient had undergone a previous resection and so the trachea was already short). This allowed additional hilar release and pulmonary ligament release. A median of 5 tracheal rings were resected (range: 3-12). One 19-year-old patient with tracheostomised subglottic stenosis was found to have destructed cricoid cartilage (Fig 2) resulting from severe chondritis. Replacement of the posterior cricoid wall was performed with costal cartilage.

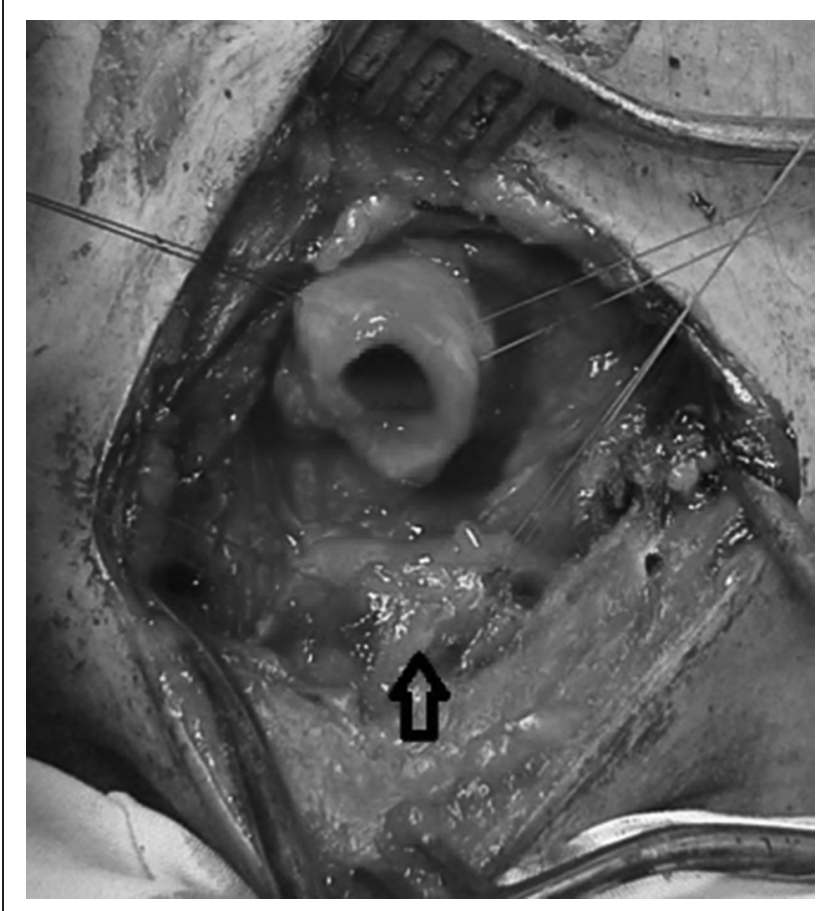

Figure 2 A 19-year-old patient with an intraoperative finding of destructed cricoid cartilage (arrow)

\section{Postoperative results}

The median length of hospital stay was 9 days (range: 7-21 days). The success rate for anastomosis was $96.7 \%(29 / 30)$. The patient with severe chondritis and cricoid replacement developed postoperative stridor. At bronchoscopy, he was found to have necrosis of the replaced cartilage and grade 3 stenosis. This patient needed a permanent tracheostomy. One patient $(3.3 \%)$ had postoperative bilateral vocal cord paralysis and a postoperative tracheostomy was inserted immediately. He was transferred to the ENT department and had a cordotomy. The tracheostomy was removed. Two further patients $(6.7 \%)$ developed unilateral vocal cord paralysis and hoarseness. In addition, two patients (6.7\%) developed a superficial wound infection. None of the eight patients undergoing first-line resection for de novo tracheal lesions suffered any morbidity.

\section{Mortality}

Our only mortality (3.3\%) was a 62 -year-old patient who had prolonged intubation for 3 weeks after suffering a neuromuscular disorder with resultant tracheal stenosis. He had multiple endoscopic dilations as he was generally unfit for surgery. He then underwent tracheal resection (including seven rings) with reanastomosis. His postoperative course was uneventful apart from limited mobility as he was wheelchair bound. On day 7, he developed a chest infection and type 1 respiratory failure. Bronchoscopy revealed a patent anastomosis and a tracheostomy was inserted as prolonged 
mechanical ventilation was expected. Two weeks later, he died of multiorgan failure.

\section{Follow-up review}

The median follow-up duration was 6 months (range: 2-21 months). Twenty-six patients were asymptomatic and very satisfied with their results after the procedure. This included the eight patients who underwent first-line resection for de novo tracheal lesions. For the two patients with unilateral vocal cord paralysis, phonation improved over the six-month follow-up period. The patient with bilateral vocal cord paralysis had no airway problems but was unsatisfied with his phonation. Those three patients were deemed mildly satisfied with their surgical results. The patient with a permanent tracheostomy was dissatisfied with his results.

\section{Discussion}

Benign postintubation tracheal stenosis is a clinical problem caused by regional ischaemic pressure necrosis of the airway. ${ }^{6}$ Stenosis can occur anywhere from the level of the ETT tip up to the glottic and subglottic area but the most common sites of stenosis are where the ETT cuff has been in contact with the tracheal wall and at the tracheal stoma site following a tracheostomy. ${ }^{7}$ As a result, tracheal stenosis occurs most commonly following the two types of airway intubation: endotracheal intubation and tracheostomy. ${ }^{8}$

The postulated causative factor for stenosis is loss or reduction of regional blood flow due to the pressure emitted through the cuff on the tracheal wall. Fortunately, the advent of large volume, low pressure cuffs has markedly reduced the occurrence of cuff related tracheal injury. ${ }^{5}$

In adults, respiratory symptoms first become apparent with exertion when the tracheal lumen (internal diameter) is reduced to approximately $50 \%$ or $8 \mathrm{~mm}$. Inspiratory stridor appears at rest when the tracheal lumen is further reduced to $5-6 \mathrm{~mm} .{ }^{9,10}$

The assessment of the degree of tracheal stenosis is an important step for each patient. Although several grading systems have been proposed, the universally accepted grading of tracheal stenosis was devised by Myer et al in 1994 (Table 1). ${ }^{11}$ The majority ( $88 \%$ ) of our patients were grade 3 or 4 and we believe this group of patients would benefit from surgery more than any other modality.

There is much evidence in the literature suggesting that for the treatment of benign postintubation tracheal stenosis,

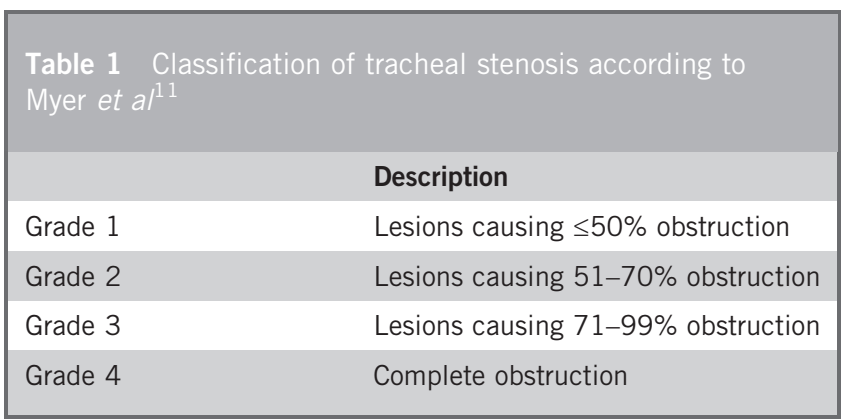

tracheal resection and anastomosis is the best available modality with regard to long-term results. ${ }^{1,2,4,12}$ Our study supports this theory but it is unique in that we attempted to perform first-line resection for tracheal stenosis whenever feasible and compared this group of patients with other patients of ours who had had previous interventions prior to resection and anastomosis.

The only patient in our series with an anastomotic complication had subglottic stenosis and cricoid chondritis. These are recognised risk factors for anastomotic complications. A retrospective review of 901 patients who underwent tracheal resection for benign or malignant stenosis (including 589 patients with postintubation tracheal stenosis) was conducted. ${ }^{13}$ Stepwise multivariate analysis revealed that prognostic factors for anastomotic complications included reoperation, diabetes mellitus, lengthy resections $(\geq 4 \mathrm{~cm})$, laryngotracheal resections, age $\leq 17$ years and the need for tracheostomy before operation.

We believe that the contraindications for tracheal resection and anastomosis are limited. In our series, no patients were refused surgery for systemic reasons. Among those patients who were refused surgery, this was mainly because of glottic stenosis where it was thought the outcome would not be favourable. Similarly, Grillo and his team opted not to perform surgery in only very few cases for systemic reasons; a more common contraindication to surgical repair of benign postintubation stenosis was the presence of very long stenosis. ${ }^{4,13}$ They performed resections with lengths ranging from $1.0 \mathrm{~cm}$ to $7.5 \mathrm{~cm}$, mostly between $2 \mathrm{~cm}$ and $4 \mathrm{~cm}$.

One of the complications in tracheal surgery is postoperative granulation tissue formation. As this occurs in proportion to the traction at the anastomosis site, choosing an appropriate type of suture is important. We prefer absorbable polydioxanone interrupted sutures. Importantly, suture tension should be appropriate and the suture knot should be formed outside the trachea to prevent formation of granulation tissue. Behrend and Klempnauer used three types of suture material (polypropylene, polydioxanone and polyglactin) in tracheal surgery in sheep. ${ }^{14}$ The results were similar in all three groups but it was noted that the suture material should be of high tensile strength and should not be absorbed in under six months. The authors concluded that the technical details (especially tension) are more important than the choice of suture material for postoperative results.

Galluccio et al proposed classifying tracheal stenosis as either simple or complex. ${ }^{15}$ They defined simple stenosis as lesions $<1 \mathrm{~cm}$ in length with no associated tracheomalacia or loss of cartilaginous support. Complex lesions were $>1 \mathrm{~cm}$ and had the greatest benefit from surgical intervention. This is significant for our study as $100 \%$ of our patients had complex lesions.

In our series, $56.7 \%$ of patients had previous endoscopic dilation. Dilation is achieved with lubricated bougies of increasing diameter applying radial pressure circumferentially to the narrowed airway. Balloon dilation is an alternative method. The flexible then rigid bronchoscope can also be used to perform blunt dissection and dilation of stenosed areas under direct vision. 
For all dilating techniques, it is crucial that the path of the true airway lumen is identified. ${ }^{16}$ It is easy (particularly when the trachea is distorted) to lose sight of the true lumen, risking airway perforation. Preoperative imaging is useful for defining patient anatomy. With the associated risk of perforation and the high chance of recurrence of stenosis, one can see that dilation alone is very rarely a definitive therapy (especially in complex, high grade stenosis) and patients will ultimately need surgical intervention.

Among the few series in the literature that showed success with endoscopic dilation for benign tracheal stenosis, there is a paper published in 2014 by Ortiz et al, who treated 18 children with repeated endoscopic tracheal dilation. ${ }^{17}$ There was no recurrence of stenosis in any of the patients (median follow-up duration: 36 months, range: 5-72 months). However, this finding may not be replicable in the adult population. First, children have more favourable results with tracheal dilation as their inflammatory response is less pronounced and there is therefore less fibrous tissue formation, meaning they have a lower risk of restenosis. Second, 12 of the 18 patients had grade 1 or 2 stenosis, which has a better prognosis with dilation than higher grade stenosis. Finally, Ortiz et al applied mitomycin $\mathrm{C}$ as an antifibroblast agent to reduce the chance of recurrence.

There is a wealth of literature supporting the use of tracheal stents in treating benign and malignant tracheal stenosis. Four patients $(13.3 \%)$ in our series went on to have tracheal stents after multiple failed dilations. The use of metallic stents in malignant stenosis is well established but treatment of benign stenosis using stents is still controversial.

There has been increasing enthusiasm for using covered self-expanding metal stents such as Ultraflex ${ }^{\mathrm{TM}}$ (Boston Scientific, Natick, MA, US), which are built from a single layer of knitted flexible nitinol with an outer covering of silicone. ${ }^{18}$ They have excellent flexibility, good biocompatibility and the self-expanding force is an important factor that enables the nitinol mesh to attach directly to the tracheal wall. While some reports have shown that there are few complications when self-expanding metal stents are used to treat benign tracheal stenosis ${ }^{19}$ others have found complications occurring in $52-87 \%$ of patients. ${ }^{20}$ These include granuloma, mucus retention and stent fracture. ${ }^{20}$ Such concerns have prompted the US Food and Drug Administration to publish a public health notification on the use of metallic stents in patients with benign airway disease. ${ }^{21}$

Martinez-Ballarin et al reported tracheal stenting with silicone Dumon ${ }^{\circledR}$ stents (Novatech, La Ciotat, France) in 64 patients with inflammatory benign tracheal stenosis, mainly following intubation or tracheostomy. ${ }^{22}$ The stents were well tolerated. Complications included migration (17.5\%), granulation $(6.3 \%)$ and airway obstruction due to mucostasis $(6.3 \%)$. One patient died during an urgent tracheotomy for mucous obstruction. Eleven patients who underwent permanent palliative silicone stenting had a mean follow-up duration of 486 days. Among 48 patients in whom tracheal silicone stents were inserted for curative restoration, stent removal was accomplished in $43.8 \%$. Among these, therapy was successful in $81.0 \%$ while restenosis after stent removal occurred in $19.0 \%$. Consequently, successful long-term curative restoration was achieved in only $35.4 \%$ of patients in whom the stent was inserted with curative intent (17/48) and in only $26.5 \%$ of all patients (17/64).

Other more rare modalities of treatment for benign postintubation tracheal stenosis have also not proved to be effective. Neodymium-doped yttrium aluminium garnet laser and cryotherapy have not been reported as being used in the treatment of postintubation tracheal stenosis except in very small series or case reports with no long-term follow-up review.

Tracheotomy tubes can cause severe stomal stenosis in the trachea or infraglottic region. The tracheostomy stoma must not be made too large, nor should a large tube be inserted into a small stoma by force. The most important reason for stenosis at the stoma site is damaged cartilage. This association between the tracheostomy tube and development of stenosis has been recognised for over 40 years. ${ }^{3}$ If stenosis occurs where a tracheostomy has been inserted to relieve postintubation proximal airway stenosis, the double level tracheal stenosis creates a complex surgical situation.

In collaboration with our ENT colleagues, we are now moving away from tracheostomy as the primary method of treatment for benign postintubation tracheal stenosis. We believe that a tracheostomy adds an additional burden to the complexity of surgery, even when not complicated by a new level of stenosis. The reason behind this is that we prefer to include the tracheostomy site in the resected tracheal segment to prevent healing with secondary granulation tissue. This can mean that even in cases with short tracheal stenosis, the length of segment to be resected can be considerable (especially if the tracheostomy is inserted at a low level), leading to a more difficult anastomosis.

Any patient developing stridor within weeks or months after prolonged intubation is referred to our centre for firstline bronchoscopy and assessment (with CT of the neck and chest prior to the procedure when appropriate). If deemed operable, dilation is carried out (if feasible) to allow endotracheal intubation and tracheal resection. Anastomosis is performed at the same setting. Postintubation tracheal stenosis is usually first diagnosed by an intensivist but then managed by a surgeon but we believe that patients with this clinical problem would always benefit from a multidisciplinary approach at diagnosis, involving thoracic surgeons, ENT surgeons, intensivists and anaesthesiologists, to provide the best possible outcome.

Few patients are referred to us with de novo lesions; most patients have other interventions before arriving at our centre. Better education of the primary and secondary healthcare sectors is needed regarding the effectiveness of surgical resection for tracheal stenosis and the more favourable results that can be achieved without other interventions prior to surgery.

\section{Conclusions}

First-line tracheal resection and primary anastomosis is a safe option for the treatment of tracheal stenosis following intubation without the need for repeated dilation. Collaboration is needed between thoracic and ENT surgeons to 
develop protocols for management of postintubation tracheal stenosis as patients may present to both specialties. Given the high rates of recurrence, we cannot see a role for balloon dilation, laser therapy or stent insertion in centres where specialised tracheal surgeons are available. Endoscopic dilation should be reserved for unfit patients or as a temporary bridge measure in non-equipped centres.

\section{References}

1. George M, Lang F, Pasche P, Monnier P. Surgical management of laryngotracheal stenosis in adults. Eur Arch Otorhinolaryngol 2005; 262 609-615

2. Marques $P$, Leal L, Spratley J et al. Tracheal resection with primary anastomosis: 10 years experience. Am J Otolaryngol 2009; 30: 415-418.

3. Pearson FG, Andrews MJ. Detection and management of tracheal stenosis following cuffed tube tracheostomy. Ann Thorac Surg 1971; 12: 359-374.

4. Grillo HC, Donahue DM, Mathisen DJ et al. Postintubation tracheal stenosis. J Thorac Cardiovasc Surg 1995; 109: 486-492.

5. Guyton D, Banner MJ, Kirby RR. High-volume, low-pressure cuffs. Chest 1991; 100: 1,076-1,081.

6. Wain JC. Post intubation tracheal stenosis. Chest Surg Clin N Am 2003; 13: 231-246.

7. Nouraei SA, Ma E, Patel A et al. Estimating the population incidence of adult post-intubation laryngotracheal stenosis. Clin Otolaryngol 2007; 32: 411-412.

8. Zias N, Chroneou A, Tabba MK et al. Post tracheostomy and post intubation tracheal stenosis: report of 31 cases and review of the literature. BMC Pulm Med 2008; 8: 18

9. Lavelle TF, Rotman HH, Weg JG. Isoflow-volume curves in the diagnosis of upper airway obstruction. Am Rev Respir Dis 1978; 117: 845-852.

10. Hobai IA, Chhangani SV, Alfille PH. Anesthesia for tracheal resection and reconstruction. Anesthesiol Clin 2012; 30: 709-730.
11. Myer CM, O'Connor DM, Cotton RT. Proposed grading system for subglottic stenosis based on endotracheal tube sizes. Ann Otol Rhinol Laryngol 1994; 103: 319-323.

12. Hassan FH, Goh BS, Kong MH et al. Tracheal resection and anastomosis: an 11 year management outcome. Rawal Med J 2013; 38: 177-180.

13. Wright CD, Grillo HC, Wain JC et al. Anastomotic complications after tracheal resection: prognostic factors and management. J Thorac Cardiovasc Surg 2004, 128: 731-739

14. Behrend M, Klempnauer J. Influence of suture material and technique on end-to-end reconstruction in tracheal surgery: an experimental study in sheep. Eur Surg Res 2001; 33: 210-216.

15. Galluccio G, Lucantoni G, Battistoni P et al. Interventional endoscopy in the management of benign tracheal stenoses: definitive treatment at long-term follow-up. Eur J Cardiothorac Surg 2009; 35: 429-433.

16. Bacon JL, Patterson CM, Madden BP. Indications and interventional options for non-resectable tracheal stenosis. J Thorac Dis 2014; 6: 258-270.

17. Ortiz R, Dominguez E, De La Torre $\mathrm{C}$ et al. Early endoscopic dilation and mitomycin application in the treatment of acquired tracheal stenosis. Eur $\mathrm{J}$ Pediatr Surg 2014; 24: 39-45.

18. Chin CS, Litle V, Yun J et al. Airway stents. Ann Thorac Surg 2008; 85 S792-S796.

19. Wu CY, Liu YH, Hsieh MJ et al. Airway stents in management of tracheal stenosis: have we improved? ANZ J Surg 2007; 77: 27-32.

20. Eller RL, Livingston WJ, Morgan CE et al. Expandable tracheal stenting for benign disease: worth the complications? Ann Otol Rhinol Laryngol 2006; 115: 247-252.

21. FDA Public Health Notification: Complications from Metallic Tracheal Stents in Patients with Benign Airway Disorders. Food and Drug Administration. http:// www.fda.gov/MedicalDevices/Safety/AlertsandNotices/PublicHealthNotifications/ ucm062115 (cited April 2016)

22. Martinez-Ballarin JI, Diaz-Jimenez JP, Castro MJ, Moy JA. Silicone stents in the management of benign tracheobronchial stenoses. Chest 1996; 109: 626-629. 